\title{
Chemoresistance is associated with Beclin-1 and PTEN expression in epithelial ovarian cancers
}

\author{
HUANCHUN YING ${ }^{1}$, DONGHUI QU ${ }^{2}$, CHUAN LIU $^{1}$, TIANSHU YING ${ }^{1}$, JING LV $^{3}$, \\ SHANSHAN JIN ${ }^{1}$ and HONGYING XU ${ }^{4}$
${ }^{1}$ Department of Gynecology and Obstetrics, Shengjing Hospital of China Medical University, Shenyang, Liaoning 110004;
${ }^{2}$ Department of Gynecology and Obstetrics, Affiliated Hospital of Chengde Medical College, Chengde, Hebei 067000; ${ }^{3}$ Department of Oncology, The Fifth Hospital of Shenyang, Shenyang, Liaoning 110023;
${ }^{4}$ Department of Gynecology and Obstetrics, The Ninth Hospital of Shenyang, Shenyang, Liaoning 10024, P.R. China

Received February 7, 2014; Accepted October 31, 2014

DOI: $10.3892 / \mathrm{ol} .2015 .2950$

\begin{abstract}
The aim of the present study was to investigate the protein expression of the autophagy-related genes, $B E C N 1$ and PTEN, and the association with drug resistance in epithelial ovarian cancers. In total, 40 patients with pathologically diagnosed epithelial ovarian cancer were divided into a chemotherapy-sensitive group $(n=20)$ and a chemotherapy-resistant group $(n=20)$, according to the results of the pre- or post-operative normative chemotherapy and the post-operative follow-up. The protein expression of the phosphatase and tensin homolog (PTEN) and the BECN1 gene product, Beclin-1, was analyzed using immunohistochemistry in the 40 patients with ovarian carcinoma. The positive rate of Beclin-1 expression was significantly lower in the resistant group (35.0\%) compared with the sensitive group (50.0\%). The positive rate of PTEN expression was also significantly lower in the resistant group (30.0\%) compared with the sensitive group $(65.0 \%)$. Furthermore, the differences in the expression rates were revealed to be significant $(\mathrm{P}<0.05)$. The expression of Beclin-1 was identified to be positively correlated with the expression of PTEN ( $\mathrm{rs}=0.816 ; \mathrm{P}<0.0001)$. The low expression of the Beclin-1 and PTEN proteins in the ovarian cancer tissues was revealed to be closely associated with drug resistance. Therefore, Beclin-1 may interact with PTEN to participate in the mechanism of drug resistance and the changes in macrophage activity observed in cases of drug-resistant ovarian cancer.
\end{abstract}

Correspondence to: Dr Huanchun Ying, Department of Gynecology and Obstetrics, Shengjing Hospital of China Medical University, 36 Sanhao Street, Shenyang, Liaoning 110004, P.R. China E-mail: tianshuyingen@163.com

Key words: Beclin-1, phosphatase and tensin homolog, ovarian cancer, platinum, resistant, autophagy

\section{Introduction}

Ovarian cancer is a gynecological malignancy with a high rate of mortality. Due to a lack of notable symptoms in the early stages, $60 \%$ of patients present with advanced-stage disease at diagnosis (1). The primary clinical treatment for ovarian cancer is cytoreductive surgery combined with platinum-based chemotherapy. Chemotherapy is a significant part of the radical treatment approach for ovarian cancers, however, platinum-based chemotherapies are prone to drug resistance; a factor that affects treatment efficacy and contributes to poor prognoses. At present, the five-year survival rate for ovarian cancer is $30-50 \%$ (2). Therefore, in order to improve the prognoses of these patients, the issue of drug-resistance must be addressed. Previous studies have identified that the abnormal regulation of autophagy is directly associated with tumorigenesis (3-5). Therefore, autophagy has become a novel target for the investigation of tumorigenesis, the inhibition of tumor growth and for determining how to overcome chemotherapy-associated drug resistance. However, the mechanism involved in the regulation of autophagy is complex.

$B E C N 1$, an autophagy-related gene located on human chromosome 17q21, encodes the protein Beclin-1, which participates in the formation of phages and can inhibit tumor growth by increasing the rate of autophagy. BECN1 has therefore been identified as a candidate tumor suppressor gene for ovarian cancer (6). Previous studies have revealed that the expression of Beclin-1 is downregulated in breast (7), prostate (8) and nasopharyngeal (9) carcinomas. Further studies have revealed that a loss of Beclin-1 expression results in reduced autophagy activity and increased tumorigenesis (10). The PTEN gene, located on chromosome 10 , is a tumor suppressor gene with dual specificity phosphatase activity. The phosphatase and tensin homolog (PTEN) protein is involved in the regulation of a variety of cellular signal transduction pathways, and is closely associated with cell growth and differentiation, and with tumorigenesis. Furthermore, PTEN is able to initiate autophagy and therefore inhibit tumor growth. In the present study, the protein expression of Beclin-1 and PTEN in ovarian cancer tissues was detected 
using immunohistochemistry. The study aimed to investigate the role of Beclin-1 and PTEN, and the association with resistance in ovarian cancer. The effect of autophagic changes in cases of drug-resistant ovarian cancer was also examined, in order to further elucidate on the mechanism of drug resistance and aid in the eventual overcoming of ovarian cancer.

\section{Materials and methods}

Patient characteristics. In total, 40 tissue samples were obtained from patients with ovarian cancer who had undergone cytoreductive surgical resection at the Shengjing Hospital of China Medical University (Shenyang, China) between January 2007 and May 2012. The cases were classified as serous carcinoma $(n=24)$, mucinous carcinoma $(n=8)$, clear cell carcinoma $(n=4)$, endometrial carcinoma $(n=2)$ or undifferentiated carcinoma $(n=2)$. The age range of the patients was 35 to 58 years old, with a median age of 48 years old. The pathological surgical staging was performed according to the 2009 International Federation of Gynecology and Obstetrics system as follows: Stage I, two cases; stage II, 10 cases; stage III, 24 cases; and stage IV, four cases (11).

The telephone or hospital examination follow-ups of the 40 cases of ovarian cancer began at diagnosis and ended on December 31,2012. The patients were pathologically diagnosed with ovarian cancer and divided into a chemotherapy-sensitive $(n=20)$ or chemotherapy-resistant $(n=20)$ group, according to the results of the pre- or post-operative normative chemotherapy and the post-operative follow-up. The grouping standards were as follows: i) Achievement of clinical remission following the initial platinum-based chemotherapy was necessary for study inclusion; and ii) cases with relapse at six months or more following the end of the chemotherapy program were taken as the chemotherapy-sensitive group, and cases with relapse within six months were used as the chemotherapy-resistant group. This study was approved by the ethics committee of Shengjing Hospital of China Medical University and written informed consent was obtained from all patients.

Immunohistochemical analysis. The Beclin-1 polyclonal rabbit (1:100 dilution) and PTEN monoclonal mouse (1:75 dilution) antigen solutions were purchased from Proteintech (Chicago, IL, USA). The immunohistochemistry Power Vision kit and 3,3'-diaminobenzidine reagent were purchased from Beijing Golden Bridge Zhongshan Biotechnology Co., Ltd. (Beijing, China).

Phosphate-buffered saline solution was used for the negative control, and normal known-positive ovarian tissue samples were used for the positive control. Using the Power Vision kit, the experimental procedure was performed according to the manufacturer's instructions. A total of 10 randomly selected high-magnification fields were analyzed under an optical microscope (AX70; Olympus Corporation, Tokyo, Japan) $48 \mathrm{~h}$ after sealing. PTEN-positive cells were identified by clear-brown granules located in the nucleus or cytoplasm, and Beclin-1-positive cells were identified by brown particles distributed throughout the cytoplasm. The percentage of positive cells to total cells was counted and awarded points according to the following criteria: $<10 \%$,
Table I. Expression of Beclin-1 and PTEN in chemoresistant and chemosensitive groups.

\begin{tabular}{|c|c|c|c|c|c|c|c|}
\hline \multirow[b]{2}{*}{ Group } & \multirow[b]{2}{*}{$\mathrm{n}$} & \multicolumn{3}{|c|}{ Beclin-1 } & \multicolumn{3}{|c|}{ PTEN } \\
\hline & & - & + & $\%$ positive & - & + & $\%$ positive \\
\hline Resistant & 20 & 13 & 7 & 35.0 & 14 & 6 & 30.0 \\
\hline Sensitive & 20 & 10 & 10 & 50.0 & 7 & 13 & 65.0 \\
\hline
\end{tabular}

PTEN, phosphatase and tensin homolog.

Table II. Association between Beclin-1 and PTEN expression.

\begin{tabular}{lrr}
\hline & \multicolumn{2}{c}{ Beclin-1 } \\
\cline { 2 - 3 } Protein & + & \\
\hline PTEN & & \\
+ & 12 & 5 \\
- & 7 & 16 \\
\hline
\end{tabular}

PTEN, phosphatase and tensin homolog.

0 points; $10-20 \%, 1$ point; $21-50 \%, 2$ points; and $>50 \%, 3$ points. The intensity of staining was also awarded points according to the following system: no color, 0 points; pale yellow, 1 point; brown, 2 points; and tan, 3 points. The total score for each case was the sum of the points for the percentage of positive cells and the staining intensity. A score of $\leq 3$ was regarded as negative for PTEN/Beclin-1 expression, whereas a score of $>3$ was regarded as positive for PTEN/Beclin-1 expression.

Statistical analysis. The statistical analysis was performed using SPSS 11.0 (SPSS, Inc., Chicago, IL, USA). The association between the clinicopathological parameters and the protein expression of Beclin-1 and PTEN was analyzed using Student's t-test. The Spearman's o test was used for the correlation analysis.

\section{Results}

In the 40 cases of ovarian cancer included in the present study, the expression of Beclin-1 was revealed to be primarily located in the cytoplasm. The positive rate of Beclin-1 expression was significantly lower in the chemotherapy-resistant group (35.0\%) compared with the chemotherapy-sensitive group $(50.0 \%)$ $(\mathrm{P}<0.05)$. The expression of PTEN was identified to be primarily located in the nucleus or cytoplasm. The positive rate of PTEN expression was significantly lower in the chemotherapy-resistant group (30.0\%) compared with the chemotherapy-sensitive group (65.0\%) $(\mathrm{P}<0.05$; Table I; Fig. 1).

The correlation analysis revealed that the intensity of Beclin-1 expression was positively correlated with the expression of PTEN in the 40 cases of ovarian cancer $(\mathrm{P}<0.05$; Table II). 

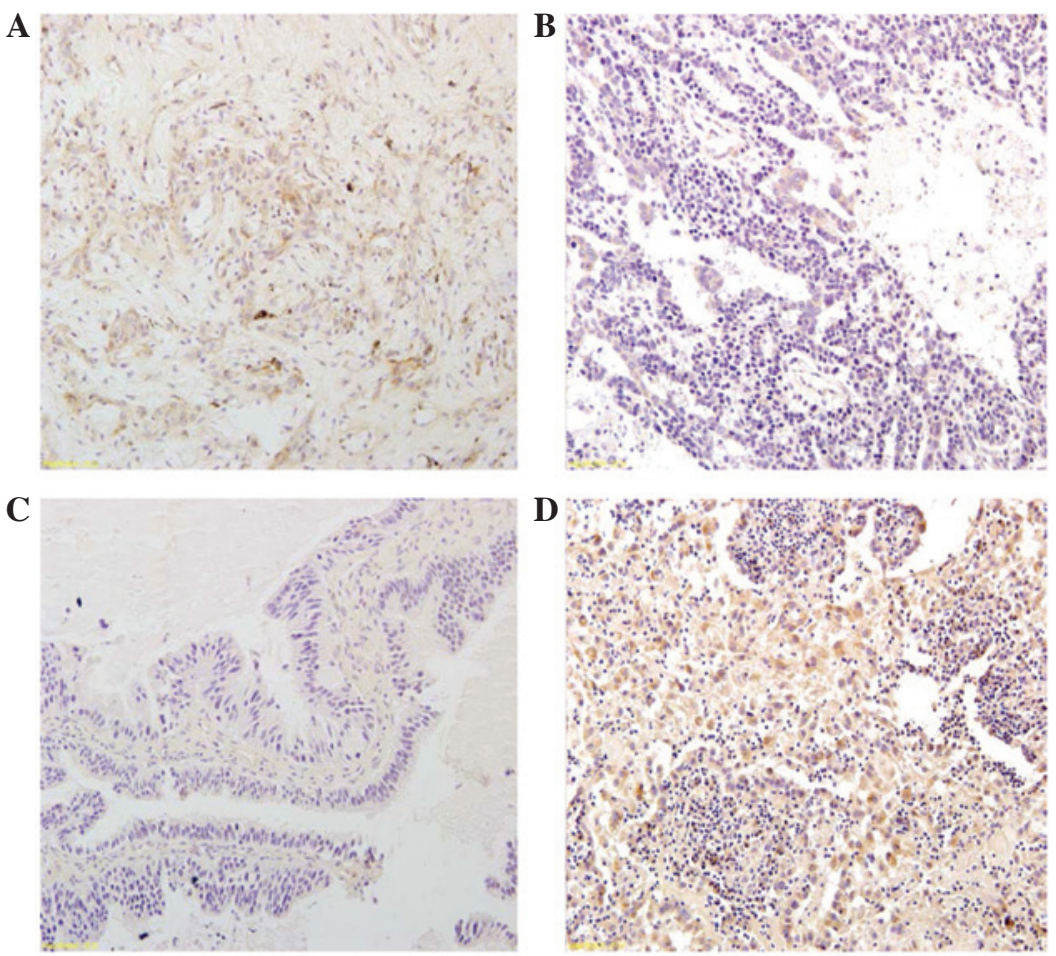

Figure 1. Immunohistochemistry revealing clear-brown nuclear and cytoplasmic granules in phosphatase and tensin homolog (PTEN)-positive cells, and brown cytoplasmic particles in Beclin-1-positive cells (magnification, x200). Chemotherapy-sensitive group; (A) PTEN and (B) Beclin-1. Chemotherapy-resistant group; (C) PTEN and (D) Beclin-1.

\section{Discussion}

Autophagy is a lysosomal degradation process for cellular macromolecules and damaged organelles, and an alternative form of programmed cell death to apoptosis in eukaryotes. Changes in the activity of signaling, transport and negative regulatory pathways of macrophages have been revealed to be associated with tumor occurrence and development. Furthermore, the abnormal expression of autophagy genes has been identified to activate or inhibit the formation of certain tumors (12).

The PTEN protein is encoded by the PTEN tumor suppressor gene located on chromosome 10 (13). Since the discovery of the gene in 1997, PTEN has gained particular attention for its role in cancer (14). Previous studies have demonstrated that the gene is associated with endometrial (15) and prostate cancers (16), and with malignant gliomas (17). According to the literature, genetic alterations in PTEN, such as mutation, loss of heterozygosity, hypermethylation, microsatellite instability or translational modifications result in the 'silencing' of gene expression (18). Previous studies revealed that the inhibition of autophagy was removed by inhibiting the change of PTEN 4,5-phosphatidylinositol diphosphate (PIP2) to 3,4,5-triphosphate phosphatidylinositol (PIP3) to participate in autophagy regulation. It has been demonstrated that PTEN and other autophagy-related genes are expressed in normal ovarian tissues, benign ovarian tumors and borderline ovarian tumors, but are downregulated in cases of ovarian cancer. This suggests that the decreased expression of autophagy-related proteins, such as PTEN, may be closely correlated with the development of ovarian cancer $(19,20)$. In addition, PTEN protein expression has been identified to be positively correlated with the differentiation state of ovarian cancers (21). A previous study demonstrated that a chemical, EF24, conferred sensitivity to drug-resistant ovarian cancer cells via the upregulation of PTEN expression (22). Furthermore, Wu et al (23) upregulated PTEN protein expression in a cisplatin-resistant ovarian cancer $\mathrm{C} 13 \mathrm{~K}$ cell line via the in vitro liposomal transfection of the PTEN gene. The C13K cells exhibited increased sensitivity to cisplatin-induced apoptosis compared with empty vector-transfected cells. An in vitro study by Yan et al (24) demonstrated that PTEN protein overexpression increased the sensitivity of the chemoresistant cell lines, CI3* and A2780cp, to cisplatin-induced apoptosis through the upregulation of $\mathrm{p} 53$, rather than the inhibition of Akt protein activation. It was further revealed that low PTEN expression was apparent in the OVCAR-3/CDDP drug-resistant ovarian cancer cell line compared with the normal OVCAR-3 ovarian cancer cell line. The phosphoinositide 3-kinase (PI3K)/Akt pathway is an important pathway involved in the regulation of autophagy, and has been revealed to be inhibited by PTEN. The study identified that PTEN protein expression was significantly lower in the drug-resistant ovarian cancer tissues compared with the drug-sensitive group. This suggested that PTEN was involved in the mechanism of drug resistance in ovarian cancer tissues via the process of autophagy.

The $\sim 150$-kb human BECN1 gene is a homolog of the atg6/vps30 yeast gene and is located on chromosome $17 \mathrm{q} 21$. $B E C N 1$ has been revealed to be involved in the development of tumors by the regulation of autophagy (25). At present, autophagy as a barrier for impeding tumorigenesis or tumor adaptive responses remains controversial (26). On the one 
hand, tumor cells are often restricted due to rapid growth, and therefore autophagy offers an adaptive response for tumor cell survival. On the other hand, certain studies have demonstrated that tumor progression is inhibited by the stimulation of non-apoptotic cell death processes, namely autophagy (27). Therefore, autophagy both inhibits tumor formation and promotes tumor development, and this association remains controversial. Recently, the dynamic role of autophagy in cancer was proposed by Kimmelman (28). The study suggested that autophagy initially acts as a barrier to prevent the initiation of tumors, but that following the formation of lesions, it positively affects malignancy and tumor maintenance. A recent study revealed that Beclin-1 expression levels differed between types of tumor cell. The expression of Beclin-1 was identified to be downregulated in a variety of tumor cells, such as those of breast, ovarian and prostate cancers, and gliomas (4). Despite this, high levels of autophagy activity were maintained in other cancer cells. In a study by Ahn et al (29), samples from 103 patients with colorectal cancer, and 60 with gastric cancer, were immunohistochemically analyzed. Beclin-1 expression was observed in 95 and $83 \%$ of the colorectal and gastric cancer samples, respectively. By contrast, little or no expression was revealed in the normal gastric and colorectal tissues. Tang et al (30) identified that the mRNA and protein expression levels of Beclin-1 were upregulated in hepatitis B virus-mediated liver cancer, which suggested a role for Beclin-1 in tumor formation. The present study revealed that Beclin-1 protein expression was significantly lower in the drug-resistant group of ovarian cancer tissues compared with the drug-sensitive group. Furthermore, the differences in expression were significant, which suggested that decreased Beclin-1 expression, and the activity of macrophages, may be associated with chemotherapy resistance and poor prognoses in patients with ovarian cancer. Conversely, an upregulation in the expression of the Beclin-1 protein may increase the sensitivity of ovarian cancers to chemotherapy, which could improve treatment efficiency and prognoses.

The present study indicated that Beclin-1 and PTEN may be co-involved in the regulation of autophagy, and therefore affect the occurrence and development of cancer. Autophagic protein turnover is regulated by type I and III PI3Ks. Type I PI3K, and the downstream signal conversion components Akt and target of rapomycin, can inhibit autophagy. PTEN induces autophagy by negative regulation of the type I PI3K. By contrast, the type III PI3K is necessary for the autophagic formation of lysosomal vacuoles. Beclin-1 regulates autophagic activity by modulating the precursor structure of Apg proteins, primarily by the formation of complexes with the type III PI3K. Therefore, Beclin-1 and PTEN may possess similar roles in the self-regulation of macrophage activity (31). The results of the present study revealed that the protein expression of Beclin-1 and PTEN exhibited a significant correlation. This indicated that the proteins may participate together in the mechanism of drug resistance observed in platinum-resistant ovarian cancers.

In summary, the protein expression of Beclin-1 and PTEN was downregulated, which suggested that a decrease in autophagic activity may be associated with drug-resistant ovarian cancers. At present, the specific mechanisms that regulate autophagy remain unclear. Further study will aid in clarifying the role of autophagy, which may provide novel solutions for treating chemotherapy-resistant ovarian cancers, and establish a reliable theoretical basis for the development of novel drugs.

The expression of the Beclin-1 and PTEN proteins, in drug-resistant and drug-sensitive ovarian cancers, was detected using immunohistochemistry and analyzed for potential correlations. The results revealed that Beclin-1 and PTEN protein expression was significantly lower in the chemotherapy-resistant group compared with the chemotherapy-sensitive group. Furthermore, the difference in expression was identified to be significant and positively correlated. The results suggested that Beclin-1 and PTEN protein expression decreased in the drug-resistant ovarian cancer tissues. Therefore, it was concluded that the occurrence of drug resistance in ovarian cancers was closely associated with a low expression of PTEN and Beclin-1. In conclusion, a reduction in autophagic activity, induced by the interaction between Beclin-1 and PTEN, may lead to drug resistance in cases of ovarian cancer.

\section{Acknowledgements}

This study was funded by the Doctor Started Natural Science Foundation (no. 20071047), the Higher Education Department Research Program (no. 2009A724), the Liaoning Science and Technology Program (no. 2010225032) and the National Natural Science Foundation of China (nos. 81372486 and 81302270).

\section{References}

1. Jemal A, Siegel R, Xu J and Ward E: Cancer statistics, 2010. CA Cancer J Clin 60: 277-300, 2010.

2. Lee S, Choi EJ, Jin C and Kim DH: Activation of PI3K/Akt pathway by PTEN reduction and PIK3CA mRNA amplification contributes to cisplatin resistance in an ovarian cancer cell line. Gynecol Oncol 97: 26-34, 2005.

3. Hara T, Nakamura K, Matsui M, et al: Suppression of basal autophagy in neural cells causes neurodegenerative disease in mice. Nature 441: 885-889, 2006.

4. Huang X, Bai HM, Chen I, Li B and Lu YC: Reduced expression of LC3B-II and Beclin 1 in glioblastoma multiforme indicates a down-regulated autophagic capacity that relates to the progression of astrocytic tumors. J Clin Neurosci 17: $1515-1519,2010$

5. Koren I and Kimchi A: Cell biology. Promoting tumorigenesis by suppressing autophagy. Science 338: 889-890, 2012

6. Lin HX, Qiu HJ, Zeng F, et al: Decreased expression of Beclin 1 correlates closely with Bcl-xL expression and poor prognosis of ovarian carcinoma. PLoS One 8: e60516, 2013.

7. Yao Q, Chen J, Lv Y, et al: The significance of expression of autophagy-related gene Beclin, Bcl-2, and Bax in breast cancer tissues. Tumour Biol 32: 1163-1171, 2011.

8. Xu LW, Qian M, Jia RP, et al: Expression and significance of microsomal prostaglandin synthase-1 (mPGES-1) and Beclin-1 in the development of prostate cancer. Asian Pac J Cancer Prev 13: 1639-1644, 2012

9. Wan XB, Fan XJ, Chen MY, et al: Elevated Beclin 1 expression is correlated with HIF-1alpha in predicting poor prognosis of nasopharyngeal carcinoma. Autophagy 6: 395-404, 2010.

10. Yue Z, Jin S, Yang C, Levine AJ and Heintz N: Beclin 1, an autophagy gene essential for early embryonic development, is a haploinsufficient tumor suppressor. Proc Natl Acad Sci USA 100: 15077-15082, 2003.

11. Pecorelli S: Revised FIGO staging for carcinoma of the vulva, cervix, and endometrium. Int J Gyneeol Obstet 105: 103-111, 2009.

12. Kondo $\mathrm{Y}$ and Kondo S: Autophagy and cancer therapy. Autophagy 2: 85-90, 2006.

13. Li DM and Sun H: TEP1, encoded by a candidate tumor suppressor locus, is a novel protein tyrosine phosphatase regulated by transforming growth factor beta. Cancer Res 57: 2124-2129, 1997. 
14. Li DM and Sun H: TEP1, encoded by a candidate tumor suppressor locus, is a novel protein tyrosine phosphatase regulated by transforming growth factor beta. Cancer Res 57: 2124-2129, 1997.

15. Lee H, Choi HJ, Kang CS, Lee HJ, Lee WS and Park CS: Expression of miRNAs and PTEN in endometrial specimens ranging from histologically normal to hyperplasia and endometrial adenocarcinoma. Mod Pathol 25: 1508-1515, 2012.

16. Patel R, Gao M, Ahmad I, Fleming J, Singh LB, Rai TS, McKie AB, Seywright M, Barnetson RJ, Edwards J, et al: Sprouty2, PTEN, and PP2A interact to regulate prostate cancer progression. J Clin Invest 123: 1157-1175, 2013.

17. Molina JR, Agarwal NK, Morales FC, Hayashi Y, Aldape KD, Cote G and Georgescu MM: PTEN, NHERF1 and PHLPP form a tumor suppressor network that is disabled in glioblastoma. Oncogene 31: 1264-1274, 2012.

18. Yang L, Kuang LG, Zheng HC, et al: PTEN encoding product: a marker for tumorigenesis and progression of gastric carcinoma. World J Gastroenterol 9: 35-39, 2003.

19. Laudański P, Kowalczuk O, Klasa-Mazurkiewicz D, et al: Selective gene expression profiling of mTOR-associated tumor suppressor and oncogenes in ovarian cancer. Folia Histochem Cytobiol 49: 317-324, 2011.

20. Skírnisdóttir I and Seidal T: Prognostic impact of concomitant p53 and PTEN on outcome in early stage (FIGO I-II) epithelial ovarian cancer. Int J Gynecol Cancer 21: 1024-1031, 2011

21. Gadducci A, Guerrieri ME and Genazzani AR: New insights on the pathogenesis of ovarian carcinoma: molecular basis and clinical implications. Gynecol Endocrinol 28: 582-586, 2012.

22. Selvendiran K, Tong L, Vishwanath S, et al: EF24 induces $\mathrm{G} 2 / \mathrm{M}$ arrest and apoptosis in cisplatin-resistant human ovarian cancer cells by increasing PTEN expression. J Biol Chem 282: 28609-28618, 2007.
23. Wu HJ, Wu HT, Weng DH, et al: Reversal of drug resistance in human ovarian cancer ceils by wild-type PTEN gene and its mechanisms. Zhonghua Fu Chan Ke Za Zhi 42: 612-616, 2007 (In Chinese).

24. Yan X, Fraser M, Qiu Q and Tsang BK: Over-expression of PTEN sensitizes human ovarian cancer cells to cisplatin-induced apoptosis in a p53-dependent manner. Gynecol Oncol 102: 348-355, 2006.

25. Scarlatti F, Maffei R, Beau I, Codogno P and Ghidoni R: Role of non-canonical Beclin 1-independent autophagy in cell death induced by resveratrol in human breast cancer cells. Cell Death Differ 15: 1318-1329, 2008.

26. Sun Y, Liu JH, Jin L, et al: Over-expression of the Beclin1 gene upregulates chemosensitivity to anti-cancer drugs by enhancing therapy-induced apoptosis in cervix squamous carcinoma CaSki cells. Cancer Lett 294: 204-210, 2010.

27. Levine B and Kroemer G: Autophagy in the pathogenesis of disease. Cell 132: 27-42, 2008.

28. Kimmelman AC: The dynamic nature of autophagy in cancer Genes Dev 25: 1999-2010, 2011.

29. Ahn CH, Jeong EG, Lee JW, et al: Expression of beclin-1, an autophagy-related protein, in gastric and colorectal cancers. APMIS 115: 1344-1349, 2007.

30. Tang H, Da L, Mao Y, et al: Hepatitis B virus X protein sensitizes cells to starvation-induced autophagy via up-regulation of beclin 1 expression. Hepatology 49: 60-71, 2009.

31. Edinger AL and Thompson CB: Defective autophagy leads to cancer. Cancer Cell 4: 422-424, 2003. 